Check for updates

Cite this: Chem. Commun., 2020,

56,7451

Received 26th March 2020

Accepted 14th May 2020

DOI: $10.1039 / \mathrm{d} 0 \mathrm{cc} 02177 f$

rsc.li/chemcomm

\section{Multi-modal control over the assembly of a molecular motor bola-amphiphile in water $\dagger$}

\author{
Fan Xu, Lukas Pfeifer, (D) Marc C. A. Stuart, (D) Franco King-Chi Leung (D) $\star_{\ddagger}$ and \\ Ben L. Feringa (D) *
}

\begin{abstract}
We report multi-modal-control over the assembly behaviour of a first-generation molecular motor bola-amphiphile in water by light, $\mathrm{pH}$ and the choice of counter-ions. These findings open up opportunities for the development of materials that reconfigurate enabling complex functions in response to different stimuli.
\end{abstract}

The variety of noncovalent interactions between assembled units found in nature provides a basis for designing artificial supramolecular systems. ${ }^{1}$ The intrinsic dynamics, tunability of properties and stimuli responsiveness of synthetic supramolecular systems is controlled by the precise design of organic molecules, ${ }^{2}$ mesogenic materials,${ }^{3}$ polymers,${ }^{4}$ and a variety of organic-inorganic hybrid composites, ${ }^{5}$ allowing modulation of, for example, assembly and functionality. ${ }^{6}$ Recent advances in supramolecular chemistry and soft materials design have enabled the creation of various supramolecular systems responsive to external stimuli, ${ }^{7}$ especially in aqueous media. ${ }^{1 a, h, 2 a, 6 d}$ Encoding specific responses to different stimuli into selfassembling systems is a promising approach to achieve more complex dynamic and adaptive behaviour. ${ }^{8}$ Besides making use of multi-stimuli responsive polymers ${ }^{4 a, 8}$ or responsive organicinorganic composites, ${ }^{5,9}$ it has also been demonstrated that supramolecular assemblies of small organic molecules in water can be controlled independently by external stimuli like light, ${ }^{10}$ $\mathrm{pH},{ }^{11}$ and ions. ${ }^{12}$ Notably, a few of these systems can be controlled by various external stimuli simultaneously. ${ }^{13}$ For example, the supramolecular assemblies of polyanionic dendritic peptide amphiphiles, reported by Besenius et al. ${ }^{13 b}$ were controlled systematically by $\mathrm{pH}$ and ionic strength. Among the various stimuli, light offers a non-invasive way with high spatial

Stratingh Institute for Chemistry, University of Groningen, Nijenborgh 4, 9747 AG, Groningen, The Netherlands. E-mail: b.l.feringa@rug.nl,

kingchifranco.leung@polyu.edu.hk

$\dagger$ Electronic supplementary information (ESI) available. See DOI: 10.1039/ d0cc02177f

\# Present address: State Key Laboratory of Chemical Biology \& Drug Discovery, Department of Applied Biology and Chemical Technology, The Hong Kong Polytechnic University, Hong Kong, China. as well as temporal precision for manipulating the structures of molecular assemblies. ${ }^{6 c, 14}$ However, implementing responsiveness to other stimuli into photo-responsive supramolecular systems based on organic molecules ${ }^{13 c}$ to realize multi-modal control over their assembly in water remains highly challenging.

Taking advantage of rotary motors as light-driven multistate switches, we have previously developed a co-assembling supramolecular system, composed of a second-generation molecular motor and a lipid (1,2-dioleoyl-sn-glycero-3-phosphocholine), allowing upon irradiation, ${ }^{15}$ transformations from nanotubes into vesicles and multi-lamellar vesicles (in a fully reversible cycle) whereby comparable packing parameters were identified for both structures $(1 / 2<P<1) .{ }^{16}$ In the present study, multimodal control over the self-assembly morphology of a firstgeneration molecular motor is reported using light, $\mathrm{pH}$ and ions as triggering elements. This class of molecular motors offers advantages due to the distinct geometric differences between cis- and trans-isomer, leading to more pronounced changes of the packing parameter upon irradiation, and the absence of thermal back-isomerization. In the present study, the morphology of the molecular motor bola-amphiphile (MBA) aggregates could be switched between sheet-like structures and a mixture of micelles and vesicles upon irradiation (Scheme 1). By contrast, morphology transitions from sheet-like assemblies

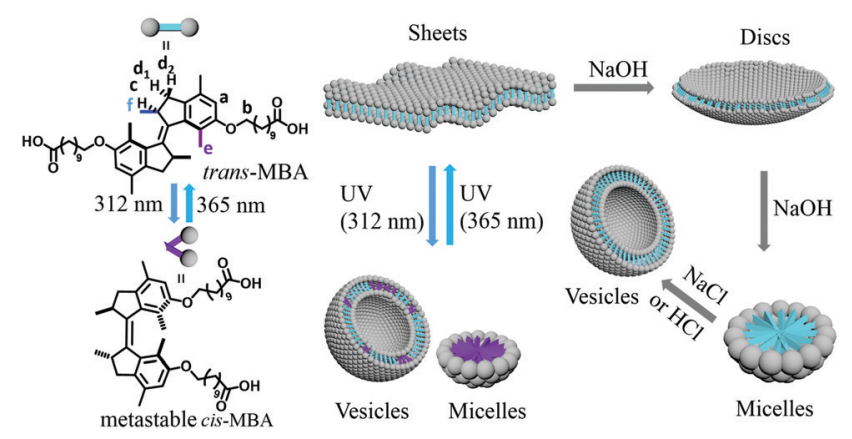

Scheme 1 Schematic illustration of the photo-isomerization of MBA and multi-modal control over its assembly behaviour. 
to discs and ultimately micelles were found with increasing $\mathrm{pH}$. Moreover, the addition of $\mathrm{NaCl}$ resulted in the formation of vesicles, while $\mathrm{CaCl}_{2}$ led to macroscopic aggregates.

The structures of trans- and cis-isomers of MBA are shown in Scheme 1. Although the rotation of first-generation motors passes through four states, ${ }^{17 a}$ the metastable trans-isomer is not discussed in this study due to its short half-life $\left(t_{1 / 2}<30 \mathrm{~s}\right)$ at room temperature. ${ }^{17 b}$ Hence, trans-MBA in this paper refers to the stable trans-isomer. Stable trans- and metastable cisisomer could be interconverted by irradiation with $312 \mathrm{~nm}$ and $365 \mathrm{~nm}$ light, respectively, while the stable cis-isomer could be obtained through thermal helix inversion from the metastable cis-isomer with a half-life of $\sim 20 \mathrm{~h} .{ }^{17 a, c}$ The stable and metastable cis-isomer are expected to have the same assembly morphology, due to their related geometry. Therefore, we mainly focus on the photo-induced morphology transition between stable trans-MBA and metastable cis-MBA in the present study. Two carboxylic acid groups are connected to the motor core through alkyl-linkers whose relative orientation significantly changes upon cis-trans isomerization, potentially leading to distinct differences in self-assembly behaviour. The carboxylic acid functionality has recently also been used as the end group on a motor amphiphile forming electrostatic interactions with counter-ions, such as $\mathrm{Ca}^{2+}$ to allow the formation of macroscopic string micro-actuators. ${ }^{10 b, 18 a}$ In the present study, we use this group as a cation binding as well as $\mathrm{pH}$ responsive unit to gain additional handles for multi-modal control over MBA's assembly behaviour in addition to photochemical cis-trans isomerization.

The synthesis and characterization of trans-MBA and stable cis-MBA are summarized in Scheme S1 (ESI $\dagger$ ). The photoresponsive behaviour was studied by UV/Vis absorption and ${ }^{1} \mathrm{H}$ NMR spectroscopy. Irradiation of the trans-isomer with $312 \mathrm{~nm}$ light resulted in a decrease of the absorption bands at $286 \mathrm{~nm}$ and $312 \mathrm{~nm}$ with concomitant formation of a new band around $350 \mathrm{~nm}$, indicating the formation of the metastable cis-isomer (Fig. S1a, ESI $\dagger$ ). After irradiating for $18 \mathrm{~min}$, no further changes were observed, meaning that the photostationary state (PSS) had been reached. Subsequent irradiation with $365 \mathrm{~nm}$ light induced the opposite spectral changes caused by the back-isomerization to trans-MBA (Fig. S1b, ESI $\dagger$ ). An isosbestic point at $329 \mathrm{~nm}$ was observed during both irradiation processes, confirming the clean formation of metastable cis- and trans-isomer, respectively. As shown in the ${ }^{1} \mathrm{H}$ NMR spectra in Fig. 1, irradiation of a sample of trans-MBA with $312 \mathrm{~nm}$ light induced the formation of a new set of signals (e.g. $H_{\mathrm{a}}=6.55 \mathrm{ppm}$, $\left.H_{\mathrm{e}}=1.21 \mathrm{ppm}\right)$ belonging to the metastable cis-isomer. The cis: trans ratio at PSS was found to be $63: 37$. Subsequent irradiation at $365 \mathrm{~nm}$ led to full recovery of the initial spectrum, demonstrating that the cis-trans isomerization of MBA can be precisely controlled by light.

To investigate the critical aggregation concentration (CAC) of trans-MBA a Nile Red fluorescence assay (NRFA), which probes the internal hydrophobicity of assemblies, ${ }^{18}$ was performed. The results revealed a CAC of $4.0 \times 10^{-6} \mathrm{M}$ for transMBA (Fig. S2a, ESI $\dagger$ ). The structure of aggregates of trans-MBA

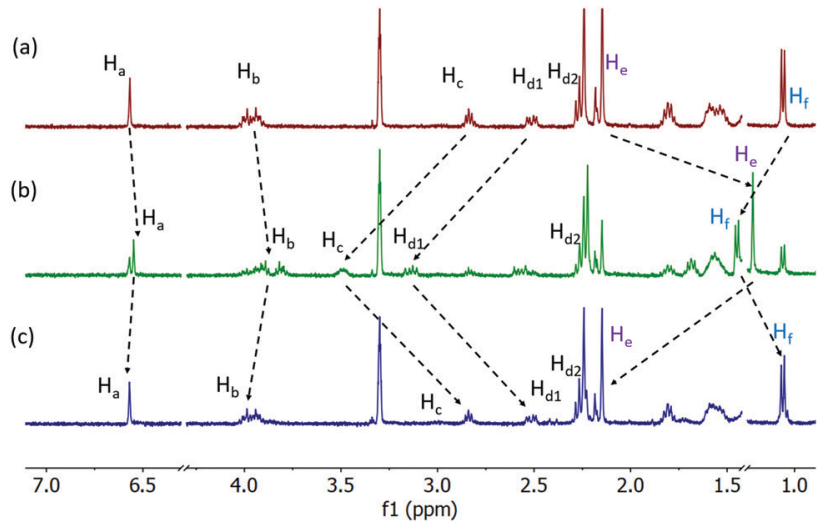

Fig. $1{ }^{1} \mathrm{H}$ NMR spectra $\left(\mathrm{CD}_{3} \mathrm{OD}, 25{ }^{\circ} \mathrm{C}, 400 \mathrm{MHz}\right)$ of trans-MBA $(2.8 \times$ $\left.10^{-3} \mathrm{M}\right)$ (a) before and (b) after irradiation to PSS with $312 \mathrm{~nm}$ light for $45 \mathrm{~min}$ at $5{ }^{\circ} \mathrm{C}$ and (c) after subsequent irradiation to PSS with $365 \mathrm{~nm}$ light for $15 \mathrm{~min}$.

in water was imaged using cryogenic transmission electron microscopy (cryo-TEM) to accurately capture their solutionstate morphology (see ESI $\dagger$ ). Since self-assembly of trans-MBA is sensitive to $\mathrm{pH}$ and ionic strength (vide infra), irradiation experiments were carried out in sodium borate buffer $(\mathrm{pH}=9.3$, $0.1 \mathrm{M}$ ). As shown in Fig. 2a, trans-MBA was found to form sheetlike assemblies $(P \approx 1)$. After irradiating with $312 \mathrm{~nm}$ light for $10 \mathrm{~min}$ to form metastable cis-MBA, vesicles with a diameter of around $20 \mathrm{~nm}$ and micelles with 5-6 nm diameter were observed in addition to sheet-like structures (Fig. 2b). As a comparison, we studied the aggregate morphology of stable cis-MBA, whose CAC $\left(1.0 \times 10^{-5} \mathrm{M}\right.$, Fig. S2b, ESI $\left.\dagger\right)$ was distinct from that of trans-MBA $\left(4.0 \times 10^{-6} \mathrm{M}\right)$, implying possible different assembly morphologies between trans- and cis-isomers. Indeed, the stable cis-isomer showed the formation of micelles with 5-6 nm diameter $(P \leq 1 / 3)$
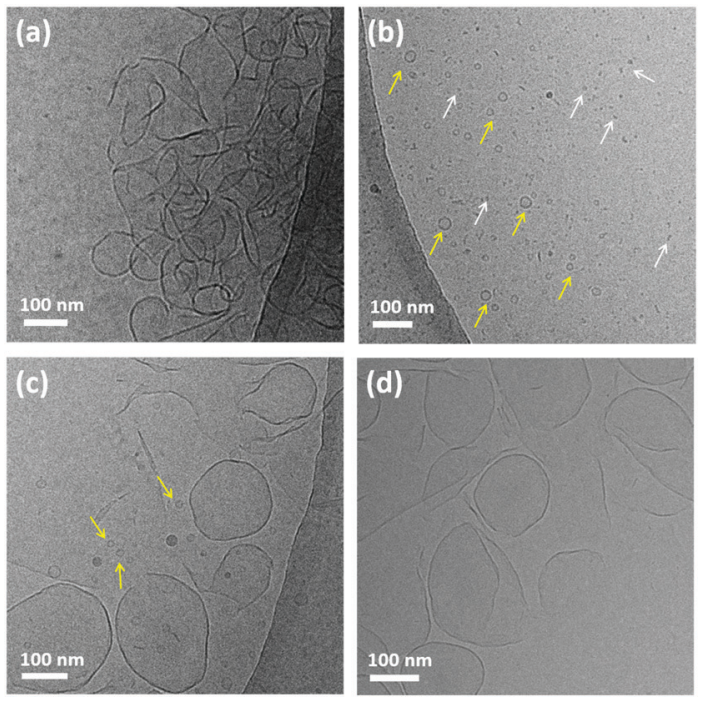

Fig. 2 Cryo-TEM images of (a) trans-MBA $\left(7.0 \times 10^{-3} \mathrm{M}\right)$ in sodium borate buffer $(\mathrm{pH}=9.3,0.1 \mathrm{M})$ before irradiation, (b) after $312 \mathrm{~nm}$ irradiation for $10 \mathrm{~min}$ and (c) after subsequent $365 \mathrm{~nm}$ irradiation for $10 \mathrm{~min}$ (d) as well as a heating-cooling cycle. (yellow: vesicles, white: micelles). 
(Fig. S4, ESI $\dagger$ ). Due to its related geometry, metastable cis-MBA is expected to have the same assembly structure as stable cis-MBA. Therefore, the presence of micelles in the sample of trans-MBA after irradiation (mixture with metastable cis-MBA) can be attributed to the self-assembly of the metastable cis isomer. The formation of vesicles $(1 / 2<P<1)$, we tentatively assigned to the co-assembly of both stable trans- and metastable cismolecules. The remaining sheet-like assemblies (Fig. S5, ESI $\dagger$ ) hint at an excess of self-assembling trans-MBA present in the mixture. This was supported by ${ }^{1} \mathrm{H}$ NMR measurement revealing a $6 \%$ share of metastable cis-MBA in the mixture (Fig. S7b, ESI $\dagger$ ). Upon subsequent irradiation with $365 \mathrm{~nm}$ light for $10 \mathrm{~min}$ (metastable cis-MBA to trans-MBA isomerization), the original sheet-like assemblies were recovered, albeit with some small vesicles remaining in the mixture (Fig. 2c). ${ }^{1} \mathrm{H}$ NMR, however, revealed that there was no remaining cis-MBA (Fig. S7c, ESI $\dagger$ ). This phenomenon had also been observed in earlier studies in our group. ${ }^{15}$ It was found that molecular motors could recover their original assembly morphologies only upon being subjected to conditions allowing them to reorganize. We therefore facilitated reorganisation by repeating the assembly preparation process (heating-cooling cycle) using the sample shown in Fig. 2c. Indeed, after this treatment, only sheet-like structures were observed (Fig. 2d).

Besides reversible control by irradiation, the assembly structure of trans-MBA also showed significant changes upon altering $\mathrm{pH}$. As shown in Fig. 3, trans-MBA formed sheet-like structures $(P \approx 1)$ at $\mathrm{pH} 8.8$ (Fig. 3a) and disc-like structures $(1 / 2<P<1)$ with a diameter of $30-40 \mathrm{~nm}$ and a thickness of 2-3 nm at pH 9.8 (Fig. 3b), as well as tiny micelles $(P \leq 1 / 3)$ with a diameter of 4-5 nm at pH 11 (Fig. 3c). Therefore, as $\mathrm{pH}$ goes up structures with increasingly smaller packing parameters are formed, which we attribute to the increasing concentration of
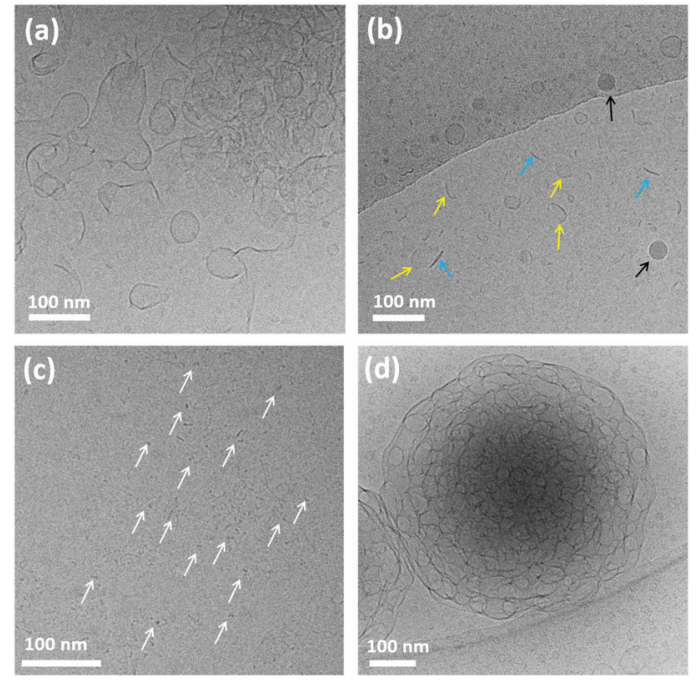

Fig. 3 Cryo-TEM images of trans-MBA $\left(7.0 \times 10^{-3} \mathrm{M}\right)$ in aq. $\mathrm{NaOH}$ solution with (a) $\mathrm{pH}=8.8$, (b) $\mathrm{pH}=9.8$, (c) $\mathrm{pH}=11$ and (d) after adjusting its $\mathrm{pH}$ to 9.4 by adding an aq. $\mathrm{HCl}$ stock solution starting from $\mathrm{pH}$ 11. (blue: upright discs, yellow: discs at an angle with the substrate, white: micelles, black: contaminants). deprotonated molecules at higher $\mathrm{pH}$ of the solution. It has been demonstrated that the head group area of long-chain fatty acids increases significantly upon dissociation of the carboxylic acid group. ${ }^{19}$ Thus, the ratio of molecules with larger head group area rises with increasing $\mathrm{pH}$. Based on the equation of molecular packing parameters for amphiphiles, ${ }^{16}$ this increase of head group area leads to a decrease of the packing parameter, causing the self-assembly to change from sheets to micelles. In our case, the morphology transformations of trans-MBA were also in accordance with this analysis. Interestingly, when trace amounts of an aq. $\mathrm{HCl}$ stock solution $(0.1 \mathrm{M})$ were added to the sample at $\mathrm{pH} 11$ to decrease the $\mathrm{pH}$ to 9.4, the morphology changed from micelles to vesicles and sponge-like structures as congeries of vesicles (Fig. 3d). In this case, however, both $\mathrm{pH}$ as well as the concentration of $\mathrm{NaCl}$ were altered and the observed transformation of morphology could be the result of changing either one or both of these conditions.

In order to explore a possible new handle for control over the assembly morphologies of trans-MBA, the counter-ion effect of $\mathrm{Na}^{+}, \mathrm{K}^{+}, \mathrm{Ca}^{2+}$, and $\mathrm{Zn}^{2+}$ on the self-assembly structures formed by trans-MBA was studied by Nile Red fluorescence assay. Therefore, the blue shift of Nile Red in trans-MBA dicarboxylate solution $\left(5.0 \times 10^{-6} \mathrm{M}\right)$ was determined in the presence of various concentrations of the chloride salts $\left(1.0 \times 10^{-6}-5.0 \times\right.$ $10^{-1} \mathrm{M}$ ) (Fig. S3, ESI $\dagger$ ). A gradual blue shift was observed with enhanced concentrations of $\mathrm{NaCl}, \mathrm{KCl}, \mathrm{ZnCl}_{2}$ and $\mathrm{CaCl}_{2}$, reflecting an increase of the internal hydrophobicity of the trans-MBA assemblies. Interestingly, the blue shift induced by increasing the concentration of $\mathrm{CaCl}_{2}$ was significantly larger compared to other chloride salts, indicating that $\mathrm{Ca}^{2+}$ ions induce aggregate formation of trans-MBA more effectively. ${ }^{18 a}$ Based on the results of NRFA, $\mathrm{Ca}^{2+}$ and $\mathrm{Na}^{+}$were chosen for additional studies of the control of assembly morphology. Vesicles with diameters of 100-200 nm were observed in aq. $0.1 \mathrm{M} \mathrm{NaCl}$ solution (Fig. 4a) contrasting with the micelles observed in samples at high $\mathrm{pH}$ discussed earlier. The addition of $\mathrm{NaCl}$ likely reduces the repulsion between the deprotonated head groups due to charge screening. ${ }^{19}$ As a consequence, the decrease in head group area results in an increase of the packing parameter of MBA leading to the observed change in assembly structure. However, in $0.1 \mathrm{M}$ aq. $\mathrm{CaCl}_{2}$ solution transMBA formed macroscopic precipitates. In order to study the assembly structure in more detail, a sample of trans-MBA
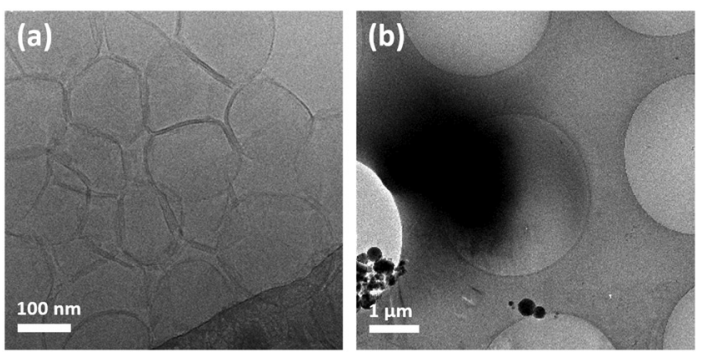

Fig. 4 Cryo-TEM images of trans-MBA dicarboxylate $\left(7.0 \times 10^{-3} \mathrm{M}\right)$ in (a) $0.1 \mathrm{M} \mathrm{NaCl}$ solution and (b) $5.0 \times 10^{-3} \mathrm{M} \mathrm{CaCl}_{2}$ solution. 
dicarboxylate at a lower $\mathrm{CaCl}_{2}$ concentration $\left(5.0 \times 10^{-3} \mathrm{M}\right)$ was characterized by cryo-TEM; micrometre-sized aggregates were observed as shown in Fig. 4b. The presence of $\mathrm{Ca}$ in these aggregates was confirmed by EDX analysis (Fig. S6, ESI $\dagger$ ). The preferential induction of aggregate formation observed for $\mathrm{Ca}^{2+}$ may be related to the stronger interaction of $\mathrm{Ca}^{2+}$ with carboxylate groups. ${ }^{18 a}$

In summary, we realized multi-modal control over the assembly behaviour of a first-generation molecular motor in water. Transitions between sheet-like structures and a mixture of micelles and vesicles, indicating large changes in packing parameter, could be controlled by light in a reversible process. Dramatic changes of assembly morphology from sheet-like assemblies to discs and ultimately micelles were achieved by adjusting $\mathrm{pH}$. Furthermore, the addition of $\mathrm{NaCl}$ led to the formation of vesicles, while $\mathrm{CaCl}_{2}$ induced macroscopic aggregates. To the best of our knowledge, this is the first example of multi-responsive assembly of a molecular motor-based bolaamphiphile in water. This study significantly enhances our understanding of the self-assembly behaviour of multiresponsive systems in water and thereby paves the way to future applications like adaptive materials, delivery systems or supramolecular materials capable to perform various distinct tasks.

This work was financially supported by the Netherlands Organization for Scientific Research (NWO-CW), the European Research Council (ERC; advanced grant no. 694345 to B. L. F.), the European Commission (MSCA-IF No. 793082 to L. P.), the Dutch Ministry of Education, Culture and Science (Gravitation program no. 024.001.035), the Chinese Scholarship Council (CSC; 201707040064) and the Croucher Foundation (Croucher Postdoctoral Fellowship Startup Allowance to F. K. C. L).

\section{Conflicts of interest}

There are no conflicts to declare.

\section{Notes and references}

1 (a) J. M. Lehn, Supramol. Chem., John Wiley \& Sons, Weinheim, 1995; (b) T. Aida, E. W. Meijer and S. I. Stupp, Science, 2012, 335, 813-817; (c) E. Krieg, M. M. C. Bastings, P. Besenius and B. Rybtchinski, Chem. Rev., 2016, 116, 2414-2477; (d) E. Mattia and S. Otto, Nat. Nanotechnol., 2015, 10, 111-119; (e) L. Voorhaar and R. Hoogenboom, Chem. Soc. Rev., 2016, 45, 4013-4031; $(f)$ F. Würthner, C. R. Saha-Möller, B. Fimmel, S. Ogi, P. Leowanawat and D. Schmidt, Chem. Rev., 2016, 116, 962-1052; $(g)$ T. F. A. De Greef, M. M. J. Smulders, M. Wolffs, A. P. H. J. Schenning, R. P. Sijbesma and E. W. Meijer, Chem. Rev., 2009, 109, 5687-5754; $(h)$ B. N. S. Thota, L. H. Urner and R. Haag, Chem. Rev., 2016, 116, 2079-2102.
2 (a) L. L. K. Taylor, I. A. Riddell and M. M. J. Smulders, Angew. Chem., Int. Ed., 2019, 58, 1280-1307; (b) J. Raeburn, A. Z. Cardoso and D. J. Adams, Chem. Soc. Rev., 2013, 42, 5143-5156.

3 (a) R. Eelkema and B. L. Feringa, Org. Biomol. Chem., 2006, 4, 3729-3745; (b) T. Kato, J. Uchida, T. Ichikawa and B. Soberats, Polym. J., 2018, 50, 149-166; (c) D. B. Amabilino, D. K. Smith and J. W. Steed, Chem. Soc. Rev., 2017, 46, 2404-2420.

4 (a) F. D. Jochum and P. Theato, Chem. Soc. Rev., 2013, 42, 7468-7483; (b) X. Yan, F. Wang, B. Zheng and F. Huang, Chem. Soc. Rev., 2012, 41, 6042-6065.

5 M. Grzelczak, L. M. Liz-Marzán and R. Klajn, Chem. Soc. Rev., 2019, 48, 1342-1361.

6 (a) O. J. G. M. Goor, S. I. S. Hendrikse, P. Y. W. Dankers and E. W. Meijer, Chem. Soc. Rev., 2017, 46, 6621-6637; (b) K. Sato, M. P. Hendricks, L. C. Palmer and S. I. Stupp, Chem. Soc. Rev., 2018, 47, 7539-7551; (c) E. Busseron, Y. Ruff, E. Moulin and N. Giuseppone, Nanoscale, 2013, 5, 7098-7140; (d) X. Ma and H. Tian, Acc. Chem. Res., 2014, 47, 1971-1981; (e) T. Muraoka and K. Kinbara, Chem. Commun., 2016, 52, 2667-2678; $(f)$ C. Heinzmann, C. Weder and L. M. de Espinosa, Chem. Soc. Rev., 2016, 45, 342-358.

7 (a) C. D. Jones and J. W. Steed, Chem. Soc. Rev., 2016, 45, 6546-6596; (b) B. L. Feringa and W. R. Browne, Molecular Switches, Wiley-VCH, Weinheim, 2011.

8 J. Zhuang, M. R. Gordon, J. Ventura, L. Li and S. Thayumanavan, Chem. Soc. Rev., 2013, 42, 7421-7435.

9 (a) O. Chovnik, R. Balgley, J. R. Goldman and R. Klajn, J. Am. Chem. Soc., 2012, 134, 19564-19567; (b) J. H. Schenkel, A. Samanta and B. J. Ravoo, Adv. Mater., 2014, 26, 1076-1080.

10 (a) W. A. Velema, M. C. A. Stuart, W. Szymanski and B. L. Feringa, Chem. Commun., 2013, 49, 5001-5003; (b) J. Chen, F. K. C. Leung, M. C. A. Stuart, T. Kajitani, T. Fukushima, E. van der Giessen and B. L. Feringa, Nat. Chem., 2018, 10, 132-138.

11 (a) T. J. Moyer, J. A. Finbloom, F. Chen, D. J. Toft, V. L. Cryns and S. I. Stupp, J. Am. Chem. Soc., 2014, 136, 14746-14752; (b) B. F. Lin, K. A. Megley, N. Viswanathan, D. V. Krogstad, L. B. Drews, M. J. Kade, Y. Qian and M. V. Tirrell, J. Mater. Chem., 2012, 22, 19447-19454.

12 S. Shin, S. Lim, Y. Kim, T. Kim, T. Choi and M. Lee, J. Am. Chem. Soc., 2013, 135, 2156-2159.

13 (a) H. A. M. Ardona and J. D. Tovar, Chem. Sci., 2015, 6, 1474-1484; (b) M. Von Gröning, I. de Feijter, M. C. A. Stuart, I. K. Voets and P. Besenius, J. Mater. Chem. B, 2013, 1, 2008-2012; (c) F. K. C. Leung, T. Kajitani, M. C. A. Stuart, T. Fukushima and B. L. Feringa, Angew. Chem., Int. Ed., 2019, 8503, 10985-10989; (d) E. Krieg, E. Shirman, H. Weissman, E. Shimoni, S. G. Wolf, I. Pinkas and B. Rybtchinski, J. Am. Chem. Soc., 2009, 131, 14365-14373.

14 S. Yagai and A. Kitamura, Chem. Soc. Rev., 2008, 37, 1520-1529.

15 D. J. van Dijken, J. Chen, M. C. A. Stuart, L. Hou and B. L. Feringa, J. Am. Chem. Soc., 2016, 138, 660-669.

$16 \mathrm{~J}$. N. Israelachvili, S. Marcelja, R. G. Horn and J. N. Israelachvili, $Q$. Rev. Biophys., 1980, 13, 121-200.

17 (a) T. van Leeuwen, G. H. Heideman, D. Zhao, S. J. Wezenberg and B. L. Feringa, Chem. Commun., 2017, 53, 6393-6396; (b) M. M. Pollard, A. Meetsma and B. L. Feringa, Org. Biomol. Chem., 2008, 6, 507-512; (c) D. Zhao, T. van Leeuwen, J. Cheng and B. L. Feringa, Nat. Chem., 2016, 9, 250-256.

18 (a) F. K. C. Leung, T. van den Enk, T. Kajitani, J. Chen, M. C. A. Stuart, J. Kuipers, T. Fukushima and B. L. Feringa, J. Am. Chem. Soc., 2018, 140, 17724-17733; (b) F. Tantakitti, J. Boekhoven, X. Wang, R. V. Kazantsev, T. Yu, J. Li, E. Zhuang, R. Zandi, J. H. Ortony, C. J. Newcomb, L. C. Palmer, G. S. Shekhawat, M. O. de la Cruz, G. C. Schatz and S. I. Stupp, Nat. Mater., 2016, 15, 469-476.

19 Y. Yan, W. Xiong, X. Li, T. Lu, J. Huang, Z. Li and H. Fu, J. Phys. Chem. B, 2007, 111, 2225-2230. 